\title{
Advances in Research on the Relationship between Vitamins and Neuropathy
}

\author{
Shengnan $\mathrm{Li}^{1}$, Sheng $\mathrm{Bi}^{1}$, Jinlian $\mathrm{Li}^{2}$, Dongmei $\mathrm{Wu}^{2}$ \\ ${ }^{1}$ Department of Neurology, The first Affiliated Hospital, Jiamusi University, Heilongjiang Jiamusi, 154000, \\ China; \\ ${ }^{2}$ School of pharmacy, Jiamusi University, Heilongjiang Jiamusi, 154000, China
}

Keywords: Vitamins, Neuropathy, Research Progress

\begin{abstract}
Neuropathy includes central and peripheral neuropathy, the pathogenesis of the disease varied, involving macrovascular and microvascular disease, metabolic factors, autoimmune mechanisms and the lack of certain growth factors in the body, In recent years, the researchers found that the level of various vitamins associated with central and peripheral neuropathy, this article on the central and peripheral neuropathy and the level of related vitamins to make a brief overview.
\end{abstract}

\section{Introduction}

The nervous system includes the central nervous system and the peripheral nervous system, central nervous system lesions mainly refers to the brain, spinal cord lesions, peripheral neuropathy, including sensory, motor and autonomic nerve lesions, and most neuropathy and diabetes disease is closely related to diabetic neuropathy is long Poor control of hyperglycemia caused by metabolic disorders in vivo, microcirculation disorders, and gradually lead to neurological ischemia, hypoxia-induced lesions, 5 to 10 years mortality rate of $25 \%-50 \%$ [1]. In recent years, the researchers found that changes in the level of various vitamins and neuropathy are closely related $[2,3]$, this article on the level of neuropathy and related vitamins to make a brief overview.

\section{Water-Soluble Vitamins}

Vitamin B6: including pyridoxine, pyridoxal and pyridoxamine, is the body of certain coenzyme composition, involved in a variety of metabolic reactions, mainly in: (1) vitamin B6 activated form of pyrophosphate phosphate can inhibit platelet aggregation , Reduce endothelial cell damage, (2) vitamin B6 to promote the conversion of linoleic acid into arachidonic acid, involved in cholesterol metabolism, lower cholesterol levels; (3) vitamin B6 is homocysteine in vivo metabolism of important coenzyme, lower blood in the same half Cystine levels. The above factors eventually lead to nerve fiber damage, and promote the occurrence and development of neuropathy. However, excessive vitamin B6 can also cause peripheral neuropathy, a recent clinical application of vitamin B6 analysis concluded that the use of vitamin B6 overdose should be closely monitored in clinical [4].

Vitamin B12: Methyl cobalamin is a biological activity of vitamin B12 coenzyme, the lack of which can lead to accumulation of tetrahydrofolate and homocysteine, both can directly damage the vascular endothelial cells, destruction of the arterial wall, causing atherosclerosis Hardening; another vitamin B12 intermediate metabolite methylmalonic acid involved in the metabolism of nerve tissue, a recent study also shows that vitamin B12 deficiency can damage neurological function, causing central and peripheral neuropathy, such as dementia, cognitive disorders, Cerebellar ataxia, peripheral neuritis and sensory disturbances, can also lead to psychiatric symptoms such as irritability, depression, etc. [5].

Vitamin B1: also known as thiamine, is one of the earliest found in vitamins, and its main phosphorylated derivative - dithiophosphoric acid (ThDP) is a necessary cofactor for glucose metabolism, and the energy source of the brain Mainly in the blood, which can affect the brain's 
energy metabolism, causing central nervous system disease; 2015, Wang D found that thiamine lack of involvement in the brain inflammatory process, including the promotion of microglia activation, CD40 / CD40L, Cytokines IL1, TNF, IL6 and arachidonic acid metabolites increased [6], and the excessive production of inflammatory mediators caused by neurotoxicity; 2017, Vignisse $\mathrm{J}$ found that thiamine deficiency can cause damage to brain function metabolism, but Thiamine precursors protect the hippocampal structure from the deleterious effects of free radicals and inflammatory factors, and play a neuroprotective role, and also play a beneficial role in neurodegenerative diseases such as Alzheimer's disease [7].

Vitamin B2: also known as riboflavin, the lack of lipid metabolism can cause the body disorders: (1) 3 - hydroxy-3-methyl-diacyl-CoA reductase (HMGR) is the in vivo cholesterol synthesis rate enzyme, high-dose Riboflavin deficiency can also reduce the activity of methylene tetrahydrofolate reductase, resulting in abnormal folic acid metabolism, leading to increased levels of homocysteine in blood (SREBPs) is an important factor involved in lipid metabolism, involved in the synthesis of fatty acids, increased homocysteine levels can cause high expression of SREBP-1c, increased triglyceride synthesis, the final Lipid metabolism disorders accelerate arteriosclerosis, causing neuropathy [8]. In addition, the clinical symptoms of riboflavin transport protein deficiency mainly involve central nervous system lesions, including cranial nerve damage (II (optic atrophy), VIII (hearing loss) and XII (tongue muscle atrophy)), sensory ataxia, eating Difficulty and breathing effort [9], but studies have shown that intake of high doses of riboflavin can improve riboflavin transport protein deficiency caused by symptoms, and the degree of improvement may be related to riboflavin dose [10].

Vitamin B9 (folic acid): folic acid is essential for the body of B water-soluble vitamins, fetal brain system in the development of indispensable; another different forms of coenzyme in a carbon unit metabolism plays an important role in the manufacture of nucleic acids, Homocysteine and involved in the process of cell growth and reproduction of important substances, folic acid levels reduced homocysteine accumulation, high concentrations of homocysteine with neurotoxicity, and the elderly brain atrophy, suffering from Parkinson's disease May be relevant. The study found that when the concentration of homocysteine increased, the rats will gradually increase growth and cognitive dysfunction, histopathology can also be found in hippocampal neurons atrophy, damage, and the nerve cells placed in high concentrations Homocysteine environmental culture will die, which is directly associated with high concentrations of homocysteine on the toxicity of nerve cells, this evidence was confirmed by Loureiro SO [11]; depression may also occur with low levels of folic acid , high levels of homocysteine, the mechanism is considered folic acid is an antioxidant that can remove oxygen free radicals, protect brain cells from free radical damage, maintain central nervous system function stability, and when folic acid Lack of hyperhomocysteinemia, oxygen free radicals increased, neuronal mitochondrial function damage, the brain antioxidant defense system imbalance, central nervous system dysfunction, causing the corresponding neuropathy and behavior abnormalities Known dysfunction [12].

Lipoic acid: is a super antioxidant, can remove, including oxygen free radicals, hydroxyl radicals, nitric oxide radicals and other free radicals, including increased glutathione (GSH) levels, Glutathione as an important antioxidant and free radical scavenger in the body, the two together inhibit the oxidative stress of nerve vessels; lipoic acid can also regenerate other antioxidants such as vitamin $C$ and vitamin $E$, in maintaining the body's entire antioxidant The balance of the system. Studies have reported that thiamine can improve cognitive function in Down's syndrome and Alzheimer's disease, a mechanism that is considered inseparable from the antioxidant function of lipoic acid [13]; secondly, in 2000, Stevens MJ and other studies have found that lipoic acid can increase the activity of $\mathrm{Na}+-\mathrm{K}+$-ATPase to improve the peripheral nerve conduction velocity in diabetic patients [14]; 2003, Coppey LJ and other diabetic rats found that lipoic acid can increase the neurotrophic blood vessels Blood flow [15]; 2004, Sekido H, etc. pointed out that long-term hyperglycemia patients, advanced glycation end products (AGEs) will be excessive production, AGEs oxidative stress can not only cause nerve fiber injury, axonal degeneration and demyelination , So that neurodegeneration, leading to neuropathy, but this process can be 
completely blocked by $\alpha$-lipoic acid [16].

\section{Fat-Soluble Vitamins}

Vitamin D Vitamin D in neuropathy in the complex mechanism, Bilir B and other diabetic peripheral neuropathy found in vitamin D and inflammatory factors IL-13, IL-17 [17], suggesting that vitamin D through the inflammatory mediator of diabetic peripheral neuropathy ; Followed by vitamin D can increase the performance of Schwann cells, play a role in the role of nutrition, myelination, repair axons and neurons, induced by nerve growth factor (NGF), to protect the peripheral nerve [18]; vitamin D has antioxidant Function, can improve the pathological changes of hippocampus in diabetic rats, while inhibiting nNOS, APP and VDR expression of overexpression, to some extent to protect the diabetic peripheral nerve from oxidative stress damage [19]; and India, a study suggests that vitamin D The lack of independent risk factors for ischemic stroke [20].

Vitamin E, Vitamin A: Vitamin A, E is an important antioxidant in the body, can remove free radicals, inhibit lipid peroxidation, improve vascular endothelial function, reduce nerve damage. In the acute phase of cerebral infarction, the body is resistant to tissue ischemia and hypoxia when the oxidative stress and free radical scavenging system disorders, the body of vitamin A, E is a large number of consumption, it is recommended that acute cerebral ischemia in vivo should be added early Vitamin A, E. Ataxia with selective vitamin E deficiency (AVED), also known as familial simple vitamin E deficiency (FIVE), is due to alpha-tocopherol transfer protein (TTPA) gene mutation leading to $\alpha$-TTP transport dysfunction In vivo changes in vitamin $\mathrm{E}$ concentration, causing symptoms of central nervous system disease, including ataxia, tendon reflexes weakened or disappeared, deep sensory disturbances and dysarthria, the experiment proved that early supplementation of high doses of vitamin E can help prevent disease progression, or even possible To reverse some neurological symptoms [21], but a meta-analysis in 2015 suggests that vitamin E supplements have no significant effect on cerebrovascular disease [22].

\section{Conclusion}

With the progress of scientific research and clinical research, vitamin deficiency caused by neuropathy, especially diabetic patients with vitamin deficiency caused by neuropathy gradually into the people's vision, vitamins have the function of diverse, together to improve or protect the nerve The role of vitamins in the neuropathy of the mechanism of action there is no more experimental basis for research, need to step further of the basic and clinical research to further explore.

\section{References}

[1] Liu selenium tellurium, Xia Ning. Diabetic peripheral neuropathy research progress [J] .Guangxi Medical University, 2010; 27 (2): 317-319

[2] He R, Hu Y, Zeng H, et al. Vitamin D deficiency increases the risk of peripheral neuropathy in Chinese patients with type 2 diabetes [J]. Diabetes Metab Res Rev, 2017, 33 (2): e2820 [3 ] Jayabalan B, Low LL.Vitamin B supplementation for diabetic peripheral. [J] .Singapore Med J. 2016, 57 (2): 55-59.

[4] Chen Min, Zhang Lingli, Quan Shuyan, etc. Vitamin B6 clinical application of the safety evaluation[J]. Chinese Journal of Pharmaceuticals. 2016, 01 (51), 65-69.

[5] Stabler SP. Vitamin B12 Deficiency [J]. N Engl J Med. 2013; 368: 149-160

[6] Spinas E, Saggini A, Kritas SK, et al. Crosstalk Between vitamin B and immunity. [J] .J Biol Regul Homeost Agents. 2015, 29 (2): 283-288.

[7] Vignisse J, Sambon M, Gorlova A, et al.Thiamine and benfotiamine prevent stress-induced suppression of hippocampal neurogenesis in mice exposed to predation without affecting brain thiamine diphosphate levels. [J] .Mol Cell Neurosci. 2017, 82: 126-136 
[8] Xin Zhonghao, Gao Weina, Guo Changjiang.Study on the Effect of Riboflavin on Lipid Metabolism [J]. Journal of Preventive Medicine of Chinese People's Liberation Army 2016, 34 (2): 281-283

[9] Foley AR, Menezes MP, Pandraud A, et al. Treated childhood neuronopathy caused by mutations in riboflavin transporter RFVT2. [J]. Brain.2014; 137 (Pt 1): 44-56

[10] Jaeger B, Bosch AM.Clinical presentation and outcome of riboflavin transporter deficiency: mini review after five years of experience. [J]. J Inherit Metab Dis. 2016; 39 (4): 559-564

[11] Loureiro SO, Heimfarth L, Pelaez Pde L, et al. Hiperhomocysteinemia developed alters expression and stoichiometry of intermediate filament and in cultured glutamate- and calcium-mediated mechanisms in rat brain during development. [J] .Int J Dev Neurosci. 2010 ; 28 (1): 21-30

[12] Kronenberg G, Colla M, Endres M. Folic acid, neurodegenerative and neuropsychiatric disease. [J]. Curr Mol Med. 2009; 9 (3): 315-323.

[13] Davidson EP, Coppey LJ, Shevalye H, et al. Impaired Corneal Sensation and Nerve Loss in a Type 2 Rat Model of Chronic Diabetes Is Reversible with Combination Therapy of Menhaden Oil, $\alpha$-Lipoic Acid, and Enalapril. . Cornea. 2017; 36 (6): 725-731.

[14] Stevens MJ, Obrosova I, Cao X, Effects of DL-alpha-lipoic acid on peripheral nerve conduction, blood flow, energy metabolism, and oxidative stress in experimental diabetic neuropathy. [J] .Diabetes. 2000; 49 (6 ): 1006-1015.

[15] Coppey LJ, Gellett JS, Davidson EP, et al. Preventing superoxide formation in epineurial arterioles of the sciatic nerve from diabetic rats restores endothelium-dependent vasodilation. [J] .Free Radic Res. 2003; 37 (1): 33 -40.

[16] Sekido H, Suzuki T, Jomori T, et al. Reduced cell replication and induction of apoptosis by advanced glycation end products in rat Schwann cells. [J]. Biochem Biophys Res Commun. 2004; 320 (1): 241- 248.

[17] Bilir B, Tulubas F, Bilir BE, et al. Association of vitamin D with inflammatory cytokines in diabetic peripheral neuropathy. [J]. Phys Phys. Ther Sci. 2016; 28 (7): 2159-2163.

[18] Nadi M, Marandi SM, Esfarjani F, et al. Comparison between Effects of 12 weeks Combined Training and Vitamin D Supplement on Improvement of Sensory-motor Neuropathy in type 2 Diabetic Women. [J] .Adv Biomed Res. 2017 , 2; 6: 55

[19] Guo F, Yue H, Wang L, et al. Vitamin D supplement ameliorates hippocampal metabolism in diabetic rats. [J]. Biochem Biophys Res Commun. 2017; 490 (2): 239-246.

[20] Chaudhuri JR, Mridula KR, Alladi S, et al.Serum 25-hydroxyvitamin d deficiency in ischemic stroke and subtypes in Indian patients. [J] .J Stroke. 2014; 16 (1): 44-50.

[21] Doria-Lamba L, De Grandis E, Cristiani E, et al. Efficacy vitamin E treatment in a child with ataxia with isolated vitamin E deficiency. [J] Eur J Pediatr. 2006; 165 (7): 494-495 The

[22] Lansing. Vitamin E supplementation has no significant effect on the occurrence of cardiovascular and cerebrovascular disease: meta-analysis of randomized controlled trials [C].2015-05-16 The Twelfth National Conference on Nutrition Science Beijing, China Xi'an Jiaotong Unive rsity Medical Department 